\title{
Promising Clinical Outcome with Long Term Follow- Up after Stereotactic Body Radiation Therapy for Elderly Patients with Oligometastatic Non-Small Cell Lung Cancer
}

\section{Xiaolong Hu}

Beijing Geriatric Hospital https://orcid.org/0000-0002-7086-5339

Hongqi Li

Air Force General Hospital PLA

Hefei Liu

Air Force General Hospital PLA

Xiaoli Kang

Air Force General Hospital PLA

\section{Xuan Wang}

Air Force General Hospital PLA

Haifeng Pang

Air Force General Hospital PLA

Chen Liu

Air Force General Hospital PLA

Jianchun Zhang

Beijing Geriatric Hospital

Yingjie Wang (D wangyj9999@126.com )

Air Force General Hospital PLA https://orcid.org/0000-0002-2646-0772

Research

Keywords: oligometastatic, elderly patients, NSCLC, radical local radiotherapy, SBRT

Posted Date: February 1st, 2021

DOI: https://doi.org/10.21203/rs.3.rs-164550/v1

License: (c) (1) This work is licensed under a Creative Commons Attribution 4.0 International License.

Read Full License 


\section{Abstract}

Objective

The ideal treatment strategy for elderly patients with metastatic non-small cell lung cancer (NSCLC) is still controversial. Our objective is to implement radical local therapy to the primary tumor and all metastases in elderly patients with oligometastatic NSCLC and evaluate its long-term efficacy and safety.

Patients and Methods

All patients were older than 65 years at initial diagnosis and had pathologically confirmed NSCLC with a total of less than or equal to 5 metastases through imaging diagnosis. Radical local therapy was applied to the primary tumor and all metastases to evaluate its OS, PFS, and toxicity.

Results

A total of 30 elderly patients with NSCLC ( $\leq 5$ metastases) received high-dose radical local radiotherapy to the primary tumor and all metastases, with a median age of 73 years (65-82 years). A total of 134 lesions were treated, with a median biologically effective dose at alpha/beta $10\left(\mathrm{BED}_{10}\right)$ value of $102 \mathrm{~Gy}$ (78-119 Gy) . The median follow-up period was 34 months (10-68 months) and the median OS was 36 months. The 1-, 2-, 3-, and 5-year OS were $93.3 \%, 66.7 \%, 49.6 \%$, and $12.1 \%$, respectively. The median PFS was 12 months, and 1-year PFS was $36.7 \%$. Multivariate analysis: When ECOG PS $<2$ and BED $10 \geq 100$ Gy, better OS was obtained $(P<0.001$ and $P=0.022)$; ECOG PS $<2$ was an independent prognostic factor for PFS ( $P=0.016)$. Most patients experienced grade 1-2 toxicities, a few experienced grade 3 toxicity $(6.66 \%)$, and no grade $4-5$ toxicities and treatment-related deaths occurred.

Conclusion

Elderly patients with oligometastatic NSCLC were given radical local radiotherapy to the primary tumor and all metastases, which improved their survival with tolerable toxicity and can be considered as an optional new treatment modality.

\section{Introduction}

Lung cancer is the malignant tumor with the highest incidence and mortality all over the world, of which $85 \%$ is non-small cell lung cancer (NSCLC) ${ }^{1}$. Approximately $40-50 \%$ of patients with NSCLC already present with distant metastases at diagnosis and $50 \%$ of them are elderly patients ${ }^{2}$. In 1995, Hellman ${ }^{3}$ proposed the concept of "oligometastasis", namely distant metastasis occurs in one or a limited number of organs, showing a target-organ effects. It occurs at the period of mild tumor invasiveness, which is the transition stage between local primary tumor and extensive metastasis. During this stage, the tumor burden is small and there is a possibility of cure. Although there is no uniform conclusion on the definition of oligometastases, recent studies ${ }^{4}$ have defined oligometastases as 3-5 metastases. 
Randomized controlled studies ${ }^{5-7}$ have shown that radical local therapy (radiotherapy or surgery) to primary tumors and metastases based on systemic therapy can significantly improve the progressionfree survival (PFS) or overall survival (OS) of patients with oligometastases and significantly improve the prognosis of patients compared with systemic therapy alone. But a systematic literature review ${ }^{8}$ reported that the five-year survival rate of patients with oligometastatic NSCLC was $8.3-86 \%$. This range is equivalent to the variation in OS of patients with stage I-IV NSCLC ${ }^{9}$. However, reports on oligometastases in the elderly are rare, and the reports on whether elderly patients with oligometastases can benefit from radical local therapy are even rarer. Elderly patients with cancer are a special group, with many underlying diseases, poor cardiopulmonary function, poor self-repair ability, decreased treatment willingness, accompanied by different degrees of geriatric syndromes, which greatly increases the difficulty of treatment and results in a poor prognosis. In this study, we retrospectively analyzed 30 elderly patients with oligometastatic NSCLC ( $\leq 5$ metastases) who were treated with radical stereotactic body radiation therapy (SBRT) to primary tumors and all metastases to evaluate the long-term efficacy and safety.

\section{Materials And Methods}

\section{Patients and Methods \\ 2.1 Patients}

A retrospective analysis was conducted on patients with oligometastatic NSCLC who were initially treated between February 2008 and October 2016 at our department. The inclusion criteria were as follows. (i) All patients were more than 65 years old. (ii) All patients were pathologically confirmed with NSCLC, and the number of metastases was $\leq 5$ excluding primary tumor and regional positive lymph nodes, by comprehensive imaging diagnosis (included but not limited to brain magnetic resonance imaging (MRI) + whole-body positron emission tomography/computed tomography (PET/CT) or brain MRI + thoracic/abdominal/pelvic CT, and bone scan was carried out when necessary). (iii) The patient had no progression of primary tumor and metastases after systemic treatment and did not develop any new metastasis for at least 2 weeks. (iv) The patient refused systemic chemotherapy but was willing to receive active treatment. (v) The patient was assessed after multidisciplinary consultation as intolerable to systemic chemotherapy but willing to be treated aggressively. (vi) The patient's underlying disease was well controlled and had qualified bone marrow hematopoietic function, liver function, and cardiopulmonary reserve. This study was approved by the Ethics Committee of Beijing Geriatric Hospital. Relevant data were approved, the patients were included in this study only after obtaining orally or written informed consent.

\subsection{Radiotherapy}

Radiotherapy planning for all elderly patients was discussed by a multidisciplinary team, including oncologists, radiation oncologists, radiologists, orthopedic surgeons, neurosurgeons, cardiologists, and respiratory physicians. The radiotherapy planning should be conducted based on patient age, cardiopulmonary function, underlying diseases, tumor location, fracture risk, central nervous system 
symptoms, and benefit-risk assessment. When the patient had obvious central nervous symptoms, local radiotherapy of intracranial metastases was performed first. If there is a high risk of vertebral fracture after consultation with orthopedic surgeons, fixation should be performed first followed by local radiotherapy. Dose fractionation mode and radiotherapy technique were shown in Table 1.

Table 1

Radiation Dose Mode

\begin{tabular}{|ll|}
\hline Sites of Disease & Treatment Regimen \\
\hline TOMO Therapy Hi.Art & \\
\hline Lung / lymph nodes & Dt $60-75$ Gy, $10-20 \mathrm{f}$ \\
\hline Bone / Adrenal & Dt $60-70$ Gy, $10-15 \mathrm{f}$ \\
\hline Thoracic/lumbar vertebra & Dt $60-70$ Gy, $10-10 \mathrm{f}$ \\
\hline Brian & Dt 40 Gy,20 f \\
\hline Spinal cord & \\
\hline Gamma-knife Radiosurgery & $70 \%$ Isodose line Dt 70-78 Gy,10-14 f \\
\hline Lung/ bone/ adrenal/ lymph nodes & $70 \%$ Isodose line Dt 70-82 Gy, 10-14 f \\
\hline Liver & \\
\hline Notes to Table 1: & \\
\hline Dt = Dose of Target & \\
\hline
\end{tabular}

\subsection{Toxicity Assessment}

Following the guidelines from the National Cancer Institute $(\mathrm{NCl})$, acute and long-term toxicities were defined before and after 90 days, using Common Terminology Criteria for Adverse Events (5th version, CTCAE). Assessed acute toxicities were dermatitis, pneumonia, fatigue, anemia, leukopenia, thrombocytopenia, and esophagitis. Long-term toxicities were pulmonary fibrosis and pleural effusion.

\subsection{Statistical Analysis}

Statistical analysis was conducted using R software(version 4.0.3 http://www.Rproject.org).Mean and standard deviation were used to describe the distribution of quantitative data, frequency and constituent ratio were used to describe the distribution of categorical data, Survival analysis was conducted, and Kaplan-Meier plot was used for calculating the survival curves. Univariable and multivariable Cox proportional hazard model were used to assess prognostic factors and calculate the survival hazard ratios (HRs) with $95 \%$ confidence interval $(95 \% \mathrm{Cl}$ ) of PFS and OS. All prognosis-related factors were included in a multivariable Cox model, regardless of their significance level of the univariate analysis. A two-sided $p$-value of $₫ 0.05$ was considered statistically significant in all aforementioned statistical tests. 


\section{Results}

\subsection{Patient Characteristics}

From February 2008 to October 2016, a total of 30 elderly patients with oligometastatic NSCLC ( $\leq 5$ metastases) received high-dose radical local radiotherapy to the primary tumor and all metastases (Fig. 1). They had a median age of 73 years (65-82 years). All patients underwent PET-CT before treatment. 11 patients received systemic chemotherapy before radiotherapy with a median chemotherapy cycle number of 2 , including 7 cases with pemetrexed + cisplatin and 4 cases with gemcitabine + cisplatin. 6 patients received systemic targeted therapy before radiotherapy, including 5 cases with Gefitinib and 1 case with Icotinib. 10 patients refused systemic chemotherapy, and 3 patients could not tolerate chemotherapy due to severe underlying diseases. A total of 134 lesions were treated with radiotherapy(irradiated metastatic sites included: 11 intracerebral metastases, 7 intrapulmonary metastases, 8 adrenal metastases, 7 intrahepatic metastases, 2 cervical lymph node metastases, 4 metastatic lesions in cervical vertebra, 10 metastatic lesions in thoracic vertebra, 11 metastatic lesions in lumbar vertebra and 4 other bone metastatic lesions), with a median $\mathrm{BED}_{10}$ of $102 \mathrm{~Gy}$ (78-119 Gy) and an average $\mathrm{BED}_{10}$ of $99.5 \mathrm{~Gy}$. Among all cases, 16 patients received radiotherapy dose $\mathrm{BED}_{10} \geq 100$ Gy to the primary tumor and all metastases. 3 patients underwent local radiotherapy for intracranial metastases due to severe central nervous symptoms, followed by radical local radiotherapy to the primary tumor and all remaining metastases 2 weeks later. 1 patient had a high risk of compression fracture due to the T8 (8th thoracic vertebra) metastasis and was transferred to the Department of Orthopaedics for surgical fixation followed by radical local radiotherapy to the primary tumor and all remaining metastases. General clinical characteristics are shown in Table 2. 
Table 2

Baseline Patient Characteristics

\begin{tabular}{|c|c|c|c|}
\hline Characteristics & $\mathbf{N}$ & No.of patients(\%) & $X \pm S$ \\
\hline \multicolumn{4}{|l|}{ Gender } \\
\hline Male & 18 & $60.0 \%$ & \\
\hline Female & 12 & $40.0 \%$ & \\
\hline $\operatorname{Age}(\mathrm{Y})$ & & & $72.9 \pm 5.35$ \\
\hline \multicolumn{4}{|l|}{ Histology } \\
\hline Adenocarcinoma & 20 & $66.7 \%$ & \\
\hline Squamous cell carcinoma & 8 & $26.7 \%$ & \\
\hline NSCLC & 2 & $6.60 \%$ & \\
\hline \multicolumn{4}{|l|}{ Mutation type } \\
\hline None & 24 & $80.0 \%$ & \\
\hline EGFR & 6 & $20.0 \%$ & \\
\hline \multicolumn{4}{|l|}{ Smoking status } \\
\hline No & 18 & $60.0 \%$ & \\
\hline Yes & 12 & $40.0 \%$ & \\
\hline \multicolumn{4}{|l|}{ Smoking Index } \\
\hline$<800$ & 17 & $56.7 \%$ & \\
\hline$\geq 800$ & 13 & $43.3 \%$ & \\
\hline \multicolumn{4}{|l|}{ ECOG PS } \\
\hline$\varangle 2$ & 17 & $56.7 \%$ & \\
\hline$\geq 2$ & 13 & $43.3 \%$ & \\
\hline \multicolumn{4}{|l|}{$\mathrm{N}$ stage } \\
\hline N0-1 & 9 & $30.0 \%$ & \\
\hline N2-3 & 21 & $70.0 \%$ & \\
\hline \multicolumn{4}{|l|}{ Comorbidity } \\
\hline No & 11 & $36.7 \%$ & \\
\hline Yes & 19 & $63.3 \%$ & \\
\hline Comorbidity type & & & \\
\hline
\end{tabular}




\begin{tabular}{|c|c|c|c|}
\hline Characteristics & $\mathbf{N}$ & No.of patients(\%) & $x \pm S$ \\
\hline None & 11 & $36.7 \%$ & \\
\hline Hypertension & 10 & $33.3 \%$ & \\
\hline Diabetes & 4 & $13.3 \%$ & \\
\hline COPD & 4 & $13.3 \%$ & \\
\hline $\mathrm{CHD}$ & 1 & $3.3 \%$ & \\
\hline \multicolumn{4}{|c|}{ Initial systemic treatment } \\
\hline No & 13 & $43.3 \%$ & \\
\hline Yes & 17 & $56.7 \%$ & \\
\hline \multicolumn{4}{|c|}{ Systemic treatment type } \\
\hline None & 13 & $43.3 \%$ & \\
\hline Chemotherapy & 11 & $36.7 \%$ & \\
\hline TKI & 6 & $20.0 \%$ & \\
\hline \multicolumn{4}{|l|}{ SBRT for $M^{*}$} \\
\hline No & 11 & $36.7 \%$ & \\
\hline Yes & 19 & $63.3 \%$ & \\
\hline \multicolumn{4}{|c|}{ CNS metastases } \\
\hline No & 21 & $70.0 \%$ & \\
\hline Yes & 9 & $30.0 \%$ & \\
\hline \multicolumn{4}{|c|}{ No.of metastases organs } \\
\hline 1 & 18 & $60.0 \%$ & \\
\hline $2-3$ & 12 & $40.0 \%$ & \\
\hline \multicolumn{4}{|c|}{ No.of metastases } \\
\hline $1-2$ & 18 & $60.0 \%$ & \\
\hline $3-5$ & 12 & $40.0 \%$ & \\
\hline \multicolumn{4}{|l|}{$\mathrm{BED}_{10}$} \\
\hline$<100 \mathrm{~Gy}$ & 14 & $46.7 \%$ & \\
\hline$\geq 100 \mathrm{~Gy}$ & 16 & $53.3 \%$ & \\
\hline
\end{tabular}




\begin{tabular}{|l|l|}
\hline Characteristics & N No.of patients(\%) $\quad \mathrm{X} \pm \mathbf{S}$ \\
\hline Notes to Table 2: & NSCLC = non-small cell lung cancer. \\
\hline Smoking Index = number of cigarettes smoked per day $x$ years of smoking. \\
\hline ECOG PS = Eastern Cooperative Oncology Group Performance Status \\
\hline COPD = Chronic Obstructive Pulmonary Disease. \\
\hline CHD = Coronary Heart Disease. \\
\hline CNS = Central Nervous System. \\
\hline BED = Biological Effective Dose. \\
\hline$M^{*}=$ Newly diagnosed metastases site after treatment \\
\hline
\end{tabular}

\subsection{Survival outcome}

The median follow-up time was 34 months (10-68 months) and the median OS was 36 months. The 1-, 2-, 3-, and 5-year OS rates were 93.3\% (95\% Cl, 84.8-100\%), 66.7\% (95\% Cl, 51.8-85.9\%), 49.6\% (95\% Cl, $34.5-71.3 \%)$, and $12.1 \%(95 \% \mathrm{Cl}, 4.32-34.2 \%)$, respectively. The median PFS was 12 months. The 1-year PFS rates was $36.7 \%(95 \% \mathrm{Cl}, 22.9-58.7 \%)$. By September 2020, 28 patients died, including 25 with tumor progression, 2 with severe pulmonary infection, and 1 with pulmonary embolism. We further observed the differences in patient sur

vival between the groups, as shown in Figs. 2A-G.

\subsection{Univariate and Multivariate Analyses on PFS and OS}

Univariate analysis suggested that good ECOG PS (Eastern Cooperative Oncology Group Performance Status) was associated with good PFS ( $\mathrm{HR}=0.412,95 \% \mathrm{Cl}: 0.192-0.877, \mathrm{P}=0.022)$. Multivariate analysis indicated that good ECOG PS $(\mathrm{HR}=0.385,95 \% \mathrm{Cl}: 0.177-0.833, \mathrm{P}=0.016)$ was an independent prognostic factor for good PFS, as shown in Table 3. 
Table 3

Factors associated with progress free surviral in univariate and multivariate analyses

\begin{tabular}{|c|c|c|c|c|c|c|}
\hline \multirow[t]{2}{*}{ Characteristics } & \multicolumn{3}{|c|}{ Univariate } & \multicolumn{3}{|c|}{ Multivariable } \\
\hline & $H R$ & $\mathrm{HR}(95 \% \mathrm{Cl})$ & $\mathbf{P}$ & HR & $\mathrm{HR}(95 \% \mathrm{Cl})$ & $\mathbf{P}$ \\
\hline Gender & 0.989 & $0.474-2.06$ & 0.977 & - & - & - \\
\hline \multicolumn{7}{|l|}{ Female vs. Male } \\
\hline Histology & & & 0.389 & - & - & - \\
\hline Adenocarcinoma.vs. SCC & 0.673 & $0.294-1.54$ & 0.347 & - & - & - \\
\hline NSCLC vs. SCC & 0.419 & $0.095-1.85$ & 0.250 & - & - & - \\
\hline Mutation type & 0.618 & $0.251-1.52$ & 0.295 & - & - & - \\
\hline \multicolumn{7}{|l|}{ EGFR vs. None } \\
\hline \multicolumn{7}{|l|}{ Smoking status } \\
\hline Yes vs. No & 0.765 & $0.363-1.61$ & 0.480 & - & - & - \\
\hline \multicolumn{7}{|l|}{ Smoking Index } \\
\hline$\geq 800$ vs. $<800$ & 1.06 & $0.515-2.20$ & 0.867 & - & - & - \\
\hline \multicolumn{7}{|l|}{ ECOG PS } \\
\hline$\otimes 2$ vs. $\geq 2$ & 0.412 & $0.192-0.877$ & 0.022 & 0.385 & $0.177-0.833$ & 0.016 \\
\hline \multicolumn{7}{|l|}{$\mathrm{N}$ stage } \\
\hline N0-1 vs. N2-3 & 0.901 & $0.410-1.97$ & 0.794 & - & - & - \\
\hline \multicolumn{7}{|l|}{ Comorbidity } \\
\hline Yes vs. No & 0.886 & $0.413-1.90$ & 0.757 & - & - & - \\
\hline
\end{tabular}

\section{Notes to Table 3:}

NSCLC $=$ non-small cell lung cancer .

SCC = Squamous Cell Carcinoma .

ECOG PS = Eastern Cooperative Oncology Group Performance Status .

CNS $=$ Central Nervous System .

$\mathrm{BED}=$ Biological Effective Dose

$M^{*}=$ Newly diagnosed metastases site after treatment 


\begin{tabular}{|c|c|c|c|c|c|c|}
\hline \multirow[t]{2}{*}{ Characteristics } & \multicolumn{3}{|c|}{ Univariate } & \multicolumn{3}{|c|}{ Multivariable } \\
\hline & HR & $\mathrm{HR}(95 \% \mathrm{Cl})$ & $\mathbf{P}$ & HR & $\mathrm{HR}(95 \% \mathrm{Cl})$ & $\mathbf{P}$ \\
\hline Yes vs. No & 0.672 & $0.321-1.41$ & 0.292 & - & - & - \\
\hline \multicolumn{7}{|l|}{ SBRT for $\mathrm{M}^{\star}$} \\
\hline Yes vs. No & 0.722 & $0.343-1.52$ & 0.392 & - & - & - \\
\hline \multicolumn{7}{|l|}{ CNS metastases } \\
\hline Yes vs. No & 0.581 & $0.257-1.32$ & 0.193 & - & - & - \\
\hline \multicolumn{7}{|c|}{ No.of metastases organs } \\
\hline 1 vs. $2-3$ & 0.735 & $0.334-1.62$ & 0.444 & - & - & - \\
\hline \multicolumn{7}{|c|}{ No.of metastases } \\
\hline $1-2$ vs. $3-5$ & 0.617 & $0.246-1.55$ & 0.304 & & & \\
\hline \multicolumn{7}{|l|}{$\mathrm{BED}_{10} \geq 100 \mathrm{~Gy}$} \\
\hline Yes vs. No & 0.524 & $0.252-1.08$ & 0.081 & 0.493 & $0.231-1.04$ & 0.065 \\
\hline $\operatorname{Age}(\mathrm{Y})$ & 0.991 & $0.916-1.07$ & 0.815 & 0.973 & $0.896-1.06$ & 0.509 \\
\hline \multicolumn{7}{|l|}{ Notes to Table 3: } \\
\hline \multicolumn{7}{|c|}{ NSCLC = non-small cell lung cancer. } \\
\hline \multicolumn{7}{|c|}{ SCC = Squamous Cell Carcinoma. } \\
\hline \multicolumn{7}{|c|}{ ECOG PS = Eastern Cooperative Oncology Group Performance Status . } \\
\hline \multicolumn{7}{|c|}{ CNS $=$ Central Nervous System. } \\
\hline \multicolumn{7}{|c|}{ BED = Biological Effective Dose. } \\
\hline
\end{tabular}

Besides, univariate analysis showed that good ECOG PS ( $H R=0.170,95 \% \mathrm{Cl}: 0.071-0.403, \mathrm{P}=0.000)$, initial systemic therapy $(H R=0.337,95 \% \mathrm{Cl}: 0.141-0.812, P=0.015)$, and $B_{10} \geq 100$ Gy $(H R=0.348$, $95 \% \mathrm{Cl}: 0.155-0.781, \mathrm{P}=0.010$ ) were associated with good OS. Multivariate analysis indicated that good ECOG PS (HR $=0.145,95 \% \mathrm{Cl}: 0.052-0.401, \mathrm{P}<0.001)$ and $\mathrm{BED}_{10} \geq 100$ Gy $(\mathrm{HR}=0.336,95 \%$ Cl: $0.132-$ $0.855, P=0.022$ ) were independent prognostic factors for good OS, as shown in Table 4. 
Table 4

Factors associated with overall survival in univariate and multivariate analyses

\begin{tabular}{|c|c|c|c|c|c|c|}
\hline \multirow[t]{2}{*}{ Characteristics } & \multicolumn{3}{|c|}{ Univariate } & \multicolumn{3}{|c|}{ Multivariable } \\
\hline & HR & $\mathrm{HR}(95 \% \mathrm{Cl})$ & $\mathbf{P}$ & HR & $\mathrm{HR}(95 \% \mathrm{Cl})$ & $\mathbf{P}$ \\
\hline Gender & 0.992 & $0.458-2.15$ & 0.985 & - & - & - \\
\hline \multicolumn{7}{|l|}{ Female vs. Male } \\
\hline Histology & & & 0.719 & - & - & - \\
\hline Adenocarcinoma vs SCC & 0.803 & $0.332-1.94$ & 0.626 & - & - & - \\
\hline NSCLC vs SCC & 1.52 & $0.344-6.74$ & 0.580 & - & - & - \\
\hline \multicolumn{7}{|l|}{ Mutation type } \\
\hline EGFR vs. None & 0.815 & $0.305-2.18$ & 0.683 & - & - & - \\
\hline \multicolumn{7}{|l|}{ Smoking status } \\
\hline Yes vs. No & 1.67 & $0.757-3.70$ & 0.203 & - & - & - \\
\hline \multicolumn{7}{|l|}{ Smoking Index } \\
\hline$\geq 800$ vs. $<800$ & 0.706 & $0.312-1.60$ & 0.404 & - & - & - \\
\hline \multicolumn{7}{|l|}{ ECOG PS } \\
\hline$\otimes 2$ vs. $\geq 2$ & 0.170 & $0.071-0.403$ & 0.000 & 0.145 & $0.052-0.401$ & $<0.001$ \\
\hline \multicolumn{7}{|l|}{$\mathrm{N}$ stage } \\
\hline N0-1 vs. N2-3 & 0.781 & $0.319-1.91$ & 0.589 & - & - & - \\
\hline \multicolumn{7}{|l|}{ Comorbidity } \\
\hline Yes vs. No & 1.251 & $0.535-2.93$ & 0.606 & - & - & - \\
\hline
\end{tabular}

Notes to Table 4:

NSCLC $=$ non-small cell lung cancer.

SCC = Squamous Cell Carcinoma .

ECOG PS = Eastern Cooperative Oncology Group Performance Status

CNS $=$ Central Nervous System .

$\mathrm{BED}=$ Biological Effective Dose

$\mathrm{M}^{*}=$ Newly diagnosed metastases site after treatment 


\begin{tabular}{|c|c|c|c|c|c|c|}
\hline \multirow[t]{2}{*}{ Characteristics } & \multicolumn{3}{|c|}{ Univariate } & \multicolumn{3}{|c|}{ Multivariable } \\
\hline & HR & $\mathrm{HR}(95 \% \mathrm{Cl})$ & $\mathbf{P}$ & HR & $\mathrm{HR}(95 \% \mathrm{Cl})$ & $\mathbf{P}$ \\
\hline Yes vs. No & 0.337 & $0.141-0.812$ & 0.015 & 0.440 & $0.154-1.26$ & 0.125 \\
\hline \multicolumn{7}{|l|}{ SBRT for $\mathrm{M}^{*}$} \\
\hline Yes vs. No & 1.31 & $0.551-3.10$ & 0.543 & - & - & - \\
\hline \multicolumn{7}{|l|}{ CNS metastases } \\
\hline Yes vs. No & 0.805 & $0.349-1.86$ & 0.610 & - & - & - \\
\hline \multicolumn{7}{|c|}{ No.of metastases organs } \\
\hline 1 vs. $2-3$ & 0.975 & $0.449-2.12$ & 0.949 & - & - & - \\
\hline \multicolumn{7}{|c|}{ No.of metastases } \\
\hline $1-2$ vs. $3-5$ & 0.802 & $0.352-1.74$ & 0.728 & & & \\
\hline \multicolumn{7}{|l|}{$\mathrm{BED}_{10} \geq 100 \mathrm{~Gy}$} \\
\hline Yes vs. No & 0.348 & $0.155-0.781$ & 0.010 & 0.336 & $0.132-0.855$ & 0.022 \\
\hline $\operatorname{Age}(Y)$ & 1.05 & $0.967-1.14$ & 0.250 & 0.967 & $0.877-1.07$ & 0.504 \\
\hline \multicolumn{7}{|l|}{ Notes to Table 4: } \\
\hline \multicolumn{7}{|c|}{ NSCLC = non-small cell lung cancer. } \\
\hline \multicolumn{7}{|c|}{ SCC = Squamous Cell Carcinoma } \\
\hline \multicolumn{7}{|c|}{ ECOG PS = Eastern Cooperative Oncology Group Performance Status } \\
\hline \multicolumn{7}{|c|}{ CNS = Central Nervous System. } \\
\hline \multicolumn{7}{|c|}{ BED = Biological Effective Dose } \\
\hline$M^{*}=$ Newly dia & ases & after treatr & & & & \\
\hline
\end{tabular}

\subsection{Toxicity}

All elderly patients completed radical local radiotherapy to the primary tumor and all metastases. Most patients experienced grade 1-2 toxicity, very few had grade 3 toxicity, no grade 4 or higher toxicity occurred, and no treatment-related death occurred. The most common acute toxicities were leukopenia (n $=15,50.0 \%$ ), of which 11 cases $(73.3 \%)$ were elderly patients who had previously undergone systemic chemotherapy. The next most common were fatigue $(n=12,40.0 \%)$, pneumonia $(n=9,30.0 \%)$, and thrombocytopenia $(n=8,26.6 \%)$. The grade $2-3$ pneumonia $(n=4)$ was only found in patients with chronic obstructive pulmonary disease (COPD). The observable long-term toxicities were pulmonary fibrosis $(n=2,6.66 \%)$ and pleural effusion $(n=1,3.33 \%)$, as shown in Table 5. 
Table 5

Acute and Long-term toxicities according to CTCAEv5.0

\begin{tabular}{|llll|}
\hline CTCAEv5.0 & $\begin{array}{l}\text { Grade 1 } \\
\text { No.(\%) }\end{array}$ & $\begin{array}{l}\text { Grade 2 } \\
\text { No.(\%) }\end{array}$ & $\begin{array}{l}\text { Grade 3 } \\
\text { No.(\%) }\end{array}$ \\
\hline Acute toxicities & & & \\
\hline Dermatitis & $4(13.3 \%)$ & $2(6.67 \%)$ & 0 \\
\hline Pneumonia* & $5(16.7 \%)$ & $3(10.0 \%)$ & $1(3.33 \%)$ \\
\hline Fatigue & $10(33.3 \%)$ & $2(6.67 \%)$ & 0 \\
\hline Anemia & $3(10.0 \%)$ & $1(3.33 \%)$ & 0 \\
\hline Leukopenia & $11(36.7 \%)$ & $3(10.0 \%)$ & $1(3.33 \%)$ \\
\hline Thrombocytopenia & $7(23.3 \%)$ & $1(3.33 \%)$ & 0 \\
\hline Esophagitis & $4(13.3 \%)$ & $1(3.33 \%)$ & 0 \\
\hline Long-term toxicities & & & \\
\hline Pulmonary fibrosis & $1(3.33 \%)$ & $1(3.33 \%)$ & 0 \\
\hline Pleural effusion & $1(3.33 \%)$ & 0 & 0 \\
\hline Notes to Table 5: & & & \\
\hline Pneumonia ${ }^{*}=$ radiation-induced pneumonitis & \\
\hline
\end{tabular}

\section{Discussion}

Generally, for elderly patients with metastases, maintaining or improving the quality of life is often the primary goal of treatment decisions, while prolonging survival is the second ${ }^{10}$. Compared with young patients, elderly patients have a higher incidence of unexpected risks and toxicities due to tumor treatment, but the treatment-related benefits are lower. It has been reported that the rates of patients receiving guideline-recommended chemotherapy treatment decreased more with increasing age than with comorbidities ${ }^{11}$. In this study, 11 patients received systemic chemotherapy before radiotherapy, with a median chemotherapy cycle number of 2. 13 patients did not receive systemic therapy, of which, 10 patients refused systemic chemotherapy (6 patients refused chemotherapy due to age) and 3 patients could not tolerate chemotherapy due to severe underlying diseases. Although elderly cancer patients have many underlying diseases, organ function decline, and decreased willingness to treat, accompanied by different degrees of geriatric syndromes, they are also a highly heterogeneous group. With the introduction of the concept of "oligometastatic disease", its milder biological characteristics affect treatment decisions, thus treatment decisions for metastases in the elderly should be individualized, multidisciplinary, and humanistic. 
The traditional view considers local radiotherapy as a palliative care for elderly patients with advanced metastatic tumors, which can effectively reduce pain and prevent the occurrence of bone-related events, rather than being applied in the treatment of oligometastatic diseases. With the development of radiotherapy technology, SBRT presents the characteristics of "high accuracy, high local control rate, and less toxic side effects", which can achieve radical cure of tumors. Previous studies ${ }^{12-14}$ have shown that SBRT is as effective as surgery in the treatment of early NSCLC and is the preferred local therapy for patients who cannot tolerate surgery or refuse surgery. Later, SBRT was applied to the local therapy of solitary metastases, and the outcomes were also satisfactory. De Rose et al. ${ }^{15}$ performed SABR on a total of 90 pulmonary oligometastases in 60 NSCLC patients, with all BED $10>100 \mathrm{~Gy}$, a 2-year LC rate of $88.9 \%$, and OS of $32.1 \pm 3.1$ months. Milano et al. ${ }^{16}$ performed SBRT in patients with adrenal oligometastases of NSCLC, and the LC rate was $74 \%$. Goodman et al. ${ }^{17}$ reported the efficacy and adverse reactions of SBRT in the treatment of 81 patients with oligo-hepatic metastases (106 lesions in total), with a radiotherapy dose of $54 \mathrm{~Gy}$ in 3 to 5 fractions. The follow-up time was 33 months, the local control rate was $94 \%$, and the survival time was 33.6 months. With the introduction of the concept of oligometastatic disease, treatment goals and strategies have changed from palliative care to improving patients' OS, PFS, avoidance of systemic therapy, and quality of life (eg, androgen-deprivation therapy in oligometastatic prostate cancer) ${ }^{18,19}$, a transition from treating isolated metastases alone to administering curative treatment to the primary tumor and all metastases. Several high-quality randomized controlled studies ${ }^{5-7}$ have shown that this change in treatment strategy can bring survival benefits to patients and significantly improve prognosis. However, the intervention timing for local therapy is still controversial, and many studies ${ }^{5-7,20}$ have reported systemic therapy followed by radical local therapy. The benefit is that it can maximize tumor shrinkage, reduce systemic tumor burden, and reduce the risk of further distant metastasis, but the difficulty is the intervention timing of radical local therapy during systemic therapy. If the intervention is too early, the tumor burden fails to reach the maximum reduction, and the local therapy-related toxicity is increased. If it is too late, it might lead to local progression and even distant metastasis due to tumor cell resistance, miss the best timing of local therapy and greatly reduce the probability of survival benefit brought to patients by the biological inertia of "oligometastatic" disease itself. Another study (NCT: 02893332) was designed to perform local therapy followed by sequential systemic therapy for the first time. The advantage of this modality is that it can reduce the tumor burden of patients as early as possible, but it increases the risk of local therapy and systemic therapy in the case of uncertain efficacy of systemic drugs. Both treatment modalities have advantages and disadvantages. According to the results of the current study, they can undoubtedly benefit patients compared with using systemic therapy alone. In this study, the median OS of a total of 17 elderly patients who were treated with systemic therapy before local radiotherapy was compared with that of the patients who did not receive initial systemic therapy (41 vs. 23 months). Univariate analysis suggested that patients with initial systemic therapy ( $\mathrm{HR}=0.337,95 \% \mathrm{Cl}: 0.141-0.812, \mathrm{P}=0.015)$ had better OS, but this may be related to that these elderly patients were unable to receive subsequent systemic therapy. 
In our study, ECOG PS $<2$ and $\mathrm{BED}_{10} \geq 100$ Gy were independent factors for survival prognosis. A good physical score indicated that their quality of life was satisfactory, nutritional intake was sufficient, and body immune function could still play a partial role, which ensured treatment continuity when the disease progressed. BED10 $\geq 100$ Gy indicated a high local control rate in hypofractionated radiotherapy lesions, which can translate into a survival benefit in elderly patients with oligometastases. Recent studies ${ }^{21-23}$ have shown that the body can produce systemic anti-tumor immune effects, known as distant effects, enhance cellular immunity, and even kill distant metastases after radiation therapy, especially SBRT irradiation to the local tumor. Recent advances in immunotherapy have led to a change in the treatment standard of advanced NSCLC, and inhibitors of programmed cell death-1 (PD-1) or its ligand PD-L1 have become an important part of first-line systemic therapy. Immunotherapy is more suitable as systemic therapy for advanced NSCLC in the elderly, and several prospective studies on immunotherapy combined with SBRT for oligometastatic NSCLC are currently ongoing, such as NCT02316002, NCT03275597, and NCT03965468.

This study has the following shortcomings. (i) The sample size is small. (ii) This study is a retrospective study that cannot replace a prospective randomized controlled study because there are many influencing factors on whether elderly patients with oligometastatic NSCLC receive radical local therapy to the primary tumor and all metastases, which are often related to the patient's general physical condition, medical compliance, economic status, tolerance of systemic therapy, physician treatment mode, etc. (iii) We did not evaluate elderly patients with comprehensive geriatric assessment (CGA) and did not further clarify which type of oligometastatic NSCLC can benefit more from radical local radiotherapy given to the primary tumor and all metastases, which could help make appropriate treatment decisions for elderly patients with oligometastatic NSCLC.

\section{Conclusion}

In conclusion, radical local radiotherapy to the primary tumor and all metastases in elderly patients with oligometastatic NSCLC can improve their survival with tolerable toxicity, which is an optional new treatment modality.

\section{Abbreviations}

NSCLCDNon-small cell lung cancer

SBRT: Stereotactic body radiation therapy

$\mathrm{BED}_{10} \mathrm{\square Biologically} \mathrm{effective} \mathrm{dose} \mathrm{at} \mathrm{alpha/beta} 10$

OS:Overall survival

PFS: Progression free survival 
ECOG PS :Eastern cooperative oncology group performance status

MRI: Magnetic resonance imaging

PET: Positron emission tomography

CT: Computed tomography

CGA: Comprehensive geriatric assessment

COPD: Chronic obstructive pulmonary disease.

PD-1: Programmed cell death-1

PD-L10Programmed cell death-ligand 1

\section{Declarations}

\section{Availability of date and materials}

The datasets used and/or analysed during the current study are available from the corresponding author on reasonable request.

\section{Acknowledgements}

Not applicable.

\section{Funding}

The authors received no financial support for the research.

\section{Competing interests}

The authors have declared no conflicts of interest.

\section{Consent for publication}

Not applicable.

\section{Author Contributions}

Xiaolong Hu prepared the manuscript and conducted the literature search; Xiaolong Hu Yingjie Wang and Hongqi Li reviewed and edited the manuscript; Hefei Liu,, Jianchun Zhang, Xuan Wang, Xiaoli Kang, Haifeng Pang and Chen Liu reviewed manuscript.

\section{Authors' information}


Department of Radiation Oncology, Beijing Geriatric Hospital, Beijing, China

Xiaolong Hu Jianchun Zhang

Department of Radiation Oncology, Air Force General Hospital, Beijing, China

Yingjie Wang Hongqi Li Hefei LiuXiaoli KangXuan WangHaifeng PangChen Liu

\section{Ethical Approval and Consent to participate}

The institutional review board (NO:BJLNYY202009) approved the study design. The patients were included in this study only after obtaining orally or written informed consent.

\section{References}

1 Siegel, R. L., Miller, K. D. \& Jemal, A. Cancer statistics, 2020. CA Cancer J Clin70, 7-30, doi:10.3322/caac.21590 (2020).

2 Bunn, P. A., Jr. \& Dimou, A. Systemic Therapy for Elderly Patients With Advanced Non-Small-Cell Lung Cancers. J Clin Onco/36, 2571-2574, doi:10.1200/jco.2018.79.2457 (2018).

3 Hellman, S. \& Weichselbaum, R. R. Oligometastases. J Clin Onco/13, 8-10, doi:10.1200/jco.1995.13.1.8 (1995).

4 Dingemans, A. C. et al. Definition of Synchronous Oligometastatic Non-Small Cell Lung Cancer-A Consensus Report. J Thorac Onco/14, 2109-2119, doi:10.1016/j.jtho.2019.07.025 (2019).

5 Gomez, D. R. et al. Local consolidative therapy versus maintenance therapy or observation for patients with oligometastatic non-small-cell lung cancer without progression after first-line systemic therapy: a multicentre, randomised, controlled, phase 2 study. The Lancet. Oncology17, 1672-1682, doi:10.1016/s1470-2045(16)30532-0 (2016).

6 lyengar, P. et al. Consolidative Radiotherapy for Limited Metastatic Non-Small-Cell Lung Cancer: A Phase 2 Randomized Clinical Trial. JAMA Onco/4, e173501, doi:10.1001/jamaoncol.2017.3501 (2018).

7 Palma, D. A. et al. Stereotactic ablative radiotherapy versus standard of care palliative treatment in patients with oligometastatic cancers (SABR-COMET): a randomised, phase 2, open-label trial. Lancet (London, England)393, 2051-2058, doi:10.1016/s0140-6736(18)32487-5 (2019).

8 Ashworth, A., Rodrigues, G., Boldt, G. \& Palma, D. Is there an oligometastatic state in non-small cell lung cancer? A systematic review of the literature. Lung Cancer82, 197-203, doi:10.1016/j.lungcan.2013.07.026 (2013).

9 Goldstraw, P. et al. The IASLC Lung Cancer Staging Project: Proposals for Revision of the TNM Stage Groupings in the Forthcoming (Eighth) Edition of the TNM Classification for Lung Cancer. $J$ Thorac 
Onco/11, 39-51, doi:10.1016/j.jtho.2015.09.009 (2016).

10 Decoster, L. et al. Geriatric Assessment and Functional Decline in Older Patients with Lung Cancer. Lung195, 619-626, doi:10.1007/s00408-017-0025-2 (2017).

11 Wang, S. et al. Impact of age and comorbidity on non-small-cell lung cancer treatment in older veterans. J Clin Onco/30, 1447-1455, doi:10.1200/jco.2011.39.5269 (2012).

12 Chang, J. Y. et al. Stereotactic ablative radiotherapy versus lobectomy for operable stage I non-smallcell lung cancer: a pooled analysis of two randomised trials. The Lancet. Oncology16, 630-637, doi:10.1016/s1470-2045(15)70168-3 (2015).

13 Li, H. et al. Promising Clinical Outcome With Long Term Follow-Up After Body Gamma Knife Stereotactic Radiosurgery for Patients With Early Stage Non-small Cell Lung Cancer. Front Onco/8, 618, doi:10.3389/fonc.2018.00618 (2018).

$14 \mathrm{Xia}, \mathrm{T}$. et al. Promising clinical outcome of stereotactic body radiation therapy for patients with inoperable Stage I/II non-small-cell lung cancer. Int J Radiat Oncol Biol Phys66, 117-125, doi:10.1016/j.jirobp.2006.04.013 (2006).

15 De Rose, F. et al. Clinical Outcome of Stereotactic Ablative Body Radiotherapy for Lung Metastatic Lesions in Non-small Cell Lung Cancer Oligometastatic Patients. Clinical oncology (Royal College of Radiologists (Great Britain))28, 13-20, doi:10.1016/j.clon.2015.08.011 (2016).

16 Milano, M. T., Katz, A. W., Zhang, H. \& Okunieff, P. Oligometastases treated with stereotactic body radiotherapy: long-term follow-up of prospective study. Int J Radiat Oncol Biol Phys83, 878-886, doi:10.1016/j.jijobp.2011.08.036 (2012).

17 Goodman, B. D., Mannina, E. M., Althouse, S. K., Maluccio, M. A. \& Cárdenes, H. R. Long-term safety and efficacy of stereotactic body radiation therapy for hepatic oligometastases. Practical radiation oncology6, 86-95, doi:10.1016/j.prro.2015.10.011 (2016).

18 Correa, R. J., Salama, J. K., Milano, M. T. \& Palma, D. A. Stereotactic Body Radiotherapy for Oligometastasis: Opportunities for Biology to Guide Clinical Management. Cancer journal (Sudbury, Mass.)22, 247-256, doi:10.1097/ppo.0000000000000202 (2016).

19 Heitmann, J. \& Guckenberger, M. Perspectives on oligometastasis: challenges and opportunities. J Thorac Dis10, 113-117, doi:10.21037/jtd.2017.12.77 (2018).

$20 \mathrm{Xu}, \mathrm{Q}$. et al. Consolidative Local Ablative Therapy Improves the Survival of Patients With Synchronous Oligometastatic NSCLC Harboring EGFR Activating Mutation Treated With First-Line EGFR-TKIs. J Thorac Onco/13, 1383-1392, doi:10.1016/j.jtho.2018.05.019 (2018). 
21 DeGregorio, M., Soe, L. \& Wolf, M. Tecemotide (L-BLP25) versus placebo after chemoradiotherapy for stage III non-small cell lung cancer (START): a randomized, double-blind, phase III trial. J Thorac Dis6, 571-573, doi:10.3978/j.issn.2072-1439.2014.05.15 (2014).

22 Giaccone, G. et al. A phase III study of belagenpumatucel-L, an allogeneic tumour cell vaccine, as maintenance therapy for non-small cell lung cancer. Eur J Cancer51, 2321-2329, doi:10.1016/j.ejca.2015.07.035 (2015).

23 Herrera, F. G., Bourhis, J. \& Coukos, G. Radiotherapy combination opportunities leveraging immunity for the next oncology practice. CA Cancer J Clin67, 65-85, doi:10.3322/caac.21358 (2017).

\section{Figures}




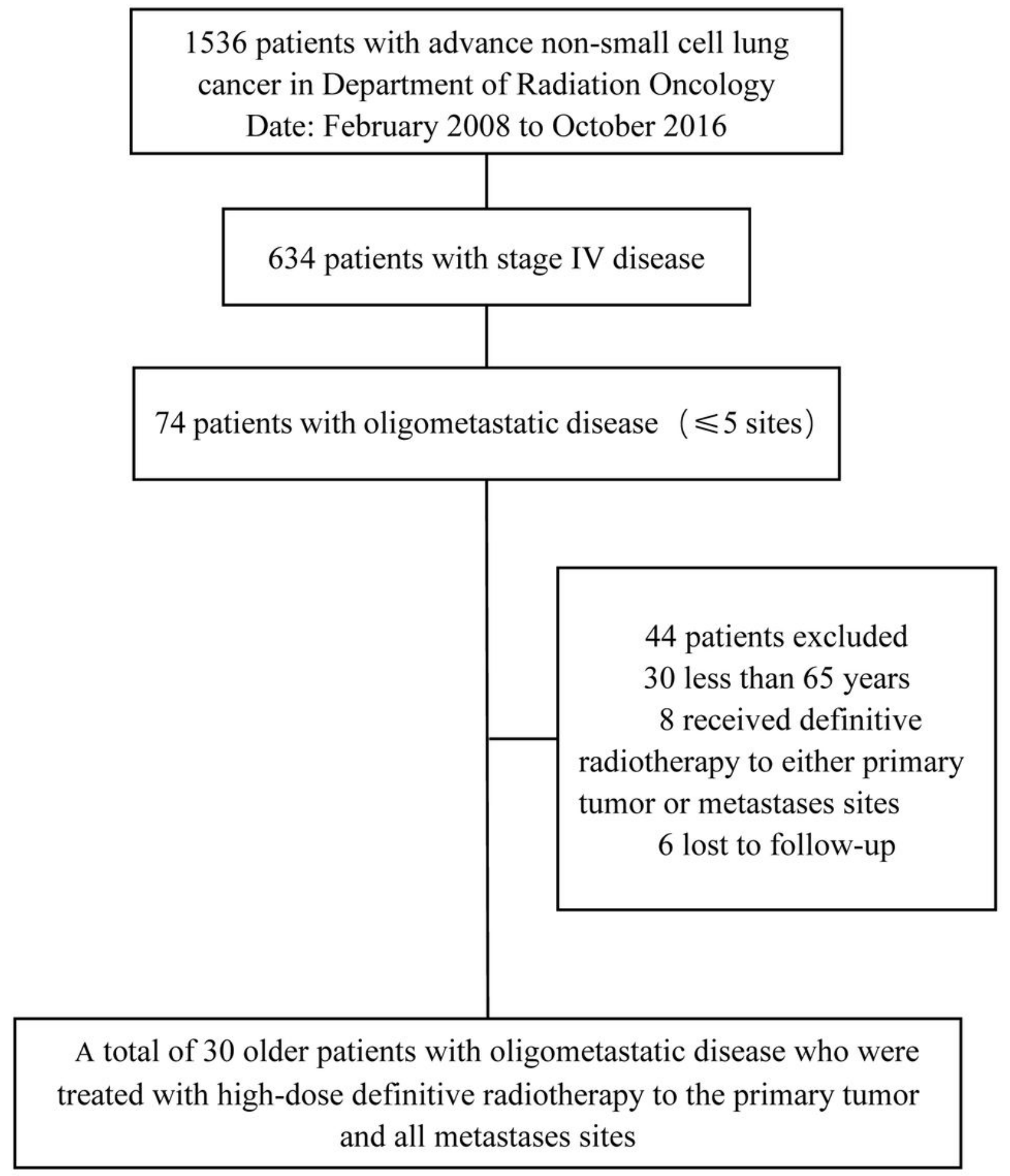

Figure 1

Flowchart of patient cohort. 

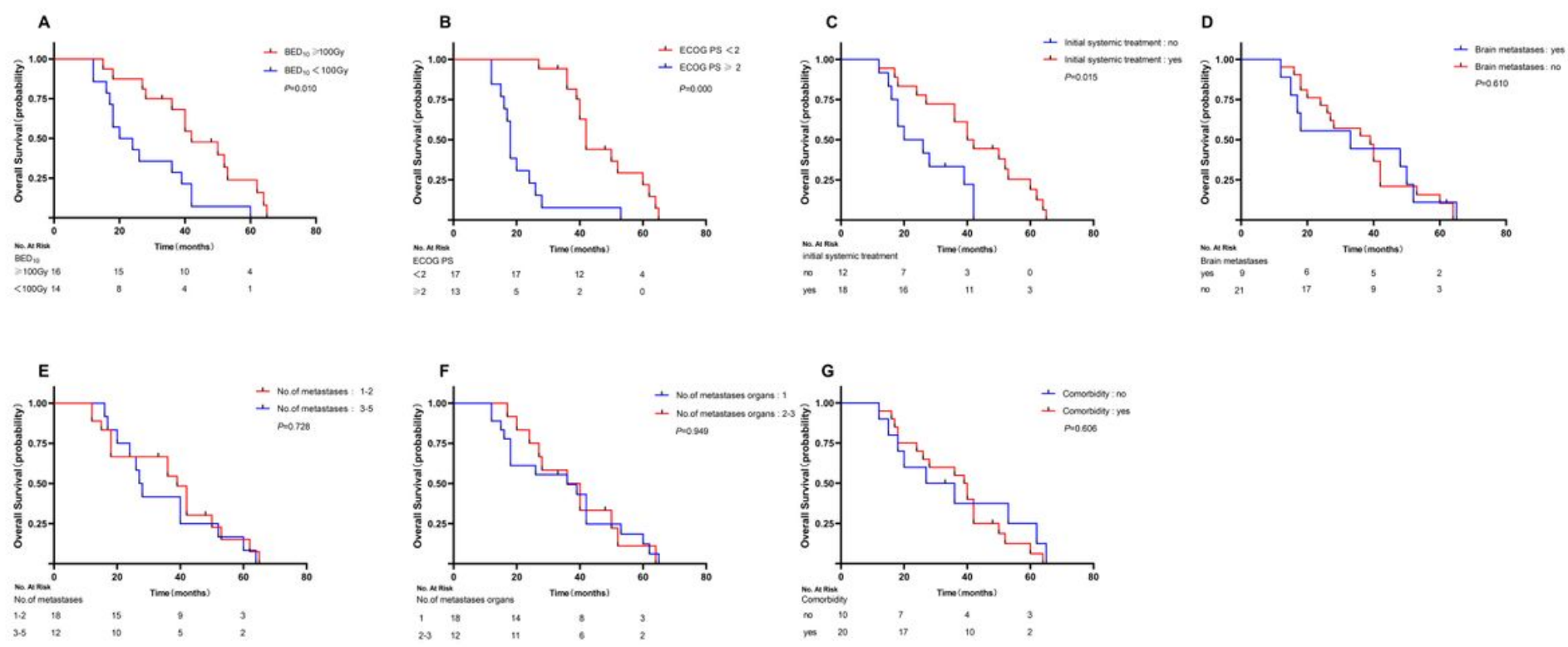

Figure 2

Kaplan-Meier curves of Overall Survival according to $\triangle A \otimes B E D 10$. (B) ECOG PS. (C) Initial systemic treatment. (D) Brain metastases. (E) No.of metastases. (F) No.of metastases organs. (G) Comorbidity. 